\title{
Phronesis
}

\section{Le tutorat par les pairs en contexte universitaire. Une étude de}

cas

\section{Tutoring in university context. A case study}

\section{Ilias-Vasileios Papaïoannou, Sofia Tsioli et Marina Vihou}

Volume 4, numéro 4, 2016

Les voies et les voix de l'accompagnement

URI : https://id.erudit.org/iderudit/1036712ar

DOI : https://doi.org/10.7202/1036712ar

Aller au sommaire du numéro

Éditeur(s)

Université de Sherbrooke

ISSN

1925-4873 (numérique)

Découvrir la revue

Citer cet article

Papaïoannou, I.-V., Tsioli, S. \& Vihou, M. (2016). Le tutorat par les pairs en contexte universitaire. Une étude de cas. Phronesis, 4(4), 46-55.

https://doi.org/10.7202/1036712ar
Résumé de l'article

Le but de l'article est de décrire une expérience éducative réalisée en contexte universitaire, afin de discuter de la contribution du tutorat par les pairs au développement de la compétence pédagogique des futurs et novices enseignants de FLE. L'étude de cas en question a duré tout au long d'un semestre académique et a impliqué des étudiants du $1^{\mathrm{er}}$ et du $2^{\mathrm{e}}$ cycle. 


\title{
Le tutorat par les pairs en contexte universitaire. Une étude de cas
}

\author{
Ilias-Vasileios PAPAÏOANNOU*, Sofia TSIOLI**, Marina VIHOU**
}

\author{
*Université d'Angers \\ CRILA \\ 11, boulevard Lavoisier \\ 49045 Cedex 01 \\ Angers, France \\ valiaspap@gmail.com \\ ** Université d'Athènes \\ Département de Langue et Littérature Françaises, Faculté des Lettres \\ Panepistimioupoli \\ 15784 Ilissia \\ Athènes, Grèce \\ sofia.tsioli@gmail.com \\ mvihou@frl.uoa.gr
}

Mots-clés : tutorat par les pairs, tuteur, tutoré, tutoriel, médiation, contexte universitaire

Résumé : Le but de l'article est de décrire une expérience éducative réalisée en contexte universitaire, afin de discuter de la contribution du tutorat par les pairs au développement de la compétence pédagogique des futurs et novices enseignants de FLE. L'étude de cas en question a duré tout au long d'un semestre académique et a impliqué des étudiants du $1^{\text {er }}$ et du $2^{e}$ cycle.

Title: Tutoring in university context. A case study

Key words: peer tutoring, tutor, tutee, tutorial, mediation, university context

Abstract: The purpose of this article is to present an educative experience carried out in a university context, in order to discuss of the ways that peer tutoring could contribute to the development of the pedagogical skills of future and novice teachers of French as a Foreign Language. The aforementioned case study lasted throughout an academic semester and involved undergraduate and graduate students. 


\section{Introduction}

Le renforcement de la compétence pédagogique des étudiants du $1^{\text {er }}$ et du $2^{\mathrm{e}}$ cycle du Département de Langue et Littérature Françaises de l'Université d'Athènes au moyen de pratiques tutorielles est à l'origine de l'expérience décrite dans cet article ${ }^{1}$. II s'agit d'examiner en temps et lieu réels les possibilités de création d'un cadre d'apprentissage autonomisant dans un contexte académique où les échanges éducatifs entre les étudiants des deux premiers cycles universitaires sont traditionnellement peu favorisés. Comment donc pourrait-on profiter du tutorat pour établir des interactions aussi bien pédagogiques que cognitives entre deux groupes d'étudiants ayant des besoins et des compétences proches, mais différents, puisque les uns en licence et les autres en master? Et comment ces échanges pourraient-ils enfin contribuer au développement de leur compétence pédagogique? Dans le but de discuter de ces questionnements, nous avons choisi comme voie d'intervention le «tutorat par les pairs» et comme champ d'action un cours de didactique, issu du rapprochement d'un cours du $1^{\text {er }}$ cycle et d'un autre du master 2.

À part l'organisation d'un nouveau cours, le rôle et les fonctions de chaque étudiant au sein de ce cours hybride ont dû être explicitement définis tout au long de l'expérience; de même pour le nouveau cadre d'apprentissage et les actions à entreprendre. Les fondements théoriques évoqués ci-dessous soulignent la relation étroite du tutorat par les pairs avec le développement de nouvelles attitudes et aptitudes. Nous présenterons également, dans les unités qui suivent les différentes étapes de réalisation de l'expérience en question ainsi que les difficultés d'application le plus souvent rencontrées. Pour discuter du degré d'efficacité de l'expérience, nous prendrons en considération non seulement les indices révélateurs de l'évolution des rapports socioaffectifs entre les participants, mais aussi les résultats obtenus lors de l'évaluation finale du cours. Les réactions des étudiants en tant que tuteurs ou tutorés repérées pendant les différentes étapes de l'expérience ainsi que leurs opinions regroupées à la fin du semestre à l'aide d'un questionnaire constitueront deux moyens supplémentaires nous permettant de discuter de l'efficacité de cette étude de cas.

\section{Enjeux théoriques et méthodologiques}

La contribution du tutorat dans l'intégration et le transfert de connaissances dans le monde du travail est depuis longtemps reconnue (Fredy-Planchot, 2007). Néanmoins, le tutorat n'a que plutôt récemment commencé à pénétrer les contextes éducatifs formels. En France, ce n'est que pendant la dernière décennie que le tutorat fait son apparition dans les universités tandis que dans les pays anglosaxons son histoire est bien plus longue et étendue (Olry-Louis, 2009, p. 77). En Grèce, le tutorat, ne faisant pas partie de la culture éducative formelle, surgit timidement dans des situations perplexes où leur caractère urgent impose le recours à des pratiques alternatives. C'est le cas, par exemple, de la gestion des élèves issus de l'immigration dont l'hétérogénéité linguistique et culturelle a imposé l'intervention des tuteurs homoglottes pour faire face à leurs besoins langagiers. Pour ce qui est du tutorat par les pairs, il est occasionnellement promu en Grèce, dans des établissements scolaires où des apprenants bilingues issus de l'immigration sont appelés à intervenir auprès de leurs camarades qui, arrivés récemment d'un autre pays, ne maîtrisent ni la langue du pays d'accueil ni la culture locale. Dans cette direction, Baudrit (2003, p.15) définit comme tutorat interculturel le processus qui consiste à "associer un élève du pays d'accueil (le tuteur) avec un

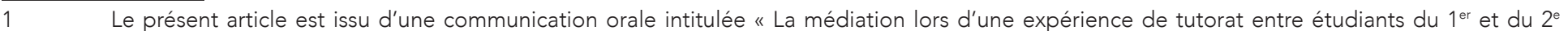

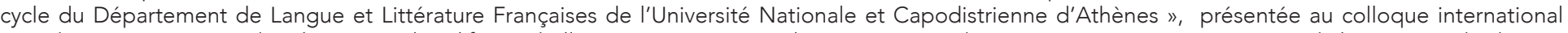

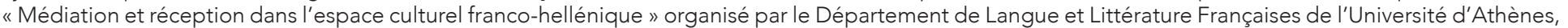
en décembre 2013.
} 
autre primo-arrivant (le tutoré)». Le tutorat est aussi appliqué comme une pratique éducative visant à intégrer des minorités socioculturelles et linguistiques, telles les enfants Roms qui rencontrent des problèmes sociocognitifs lors de leur scolarisation². Ainsi, les apprenants-tuteurs bénéficient de cette expérience de médiation pour inverser un profil identitaire (caractéristiques linguistiques et culturelles différentes de celles de la langue et culture dominantes) jusqu'alors discriminatoire.

De tout ce qui précède, nous pouvons constater que le tutorat, en tant que conduite pédagogique, est censé répondre aux besoins spécifiques d'un apprenant ou d'un groupe d'apprenants d'ordre cognitif, socioaffectif, motivationnel et métacognitif (Rodet, 2007). II s'agit alors d'une procédure pédagogique, mais aussi d'un processus social qui, nourris par les échanges interindividuels, favorisent graduellement l'autonomie des performances de l'apprenant, tout en atténuant les différences ou déviances comportementales (Becker, 1985).

En nous appuyant sur les constatations ci-dessus, nous avons voulu examiner comment cette pratique issue du monde du travail et utilisée le plus souvent pour la gestion de situations compliquées et difficiles pourrait être appliquée dans le contexte universitaire et, plus particulièrement, lors de la formation initiale et continue des enseignants de FLE. Ce transfert aurait comme objectif l'élargissement des expériences pratiques et réflexives des futurs et novices enseignants de FLE (Moussay, Étienne et Méard, 2009).

Dans cette perspective, nous avons organisé une action tutorielle impliquant des étudiants de niveau de connaissances différent. C'est cet écart, d'ailleurs, entre le niveau de connaissances des étudiants du $1^{\text {er }}$ cycle (3 ans d'études) et celui des étudiants du $2^{\mathrm{e}}$ cycle (5 ans d'études), qui nous a justement permis d'entreprendre une expérience de «médiation de proximité»(Papi, 2013, p.9), dans une tentative de rapprocher les différents savoirs des tuteurs et des tutorés impliqués. II est vrai qu'une action tutorielle met le plus souvent en avant le besoin du tutoré de progresser dans son apprentissage. II ne faudrait pas pour autant oublier que le tutorat peut s'avérer bénéfique aussi bien pour les tutorés que pour les tuteurs (Vandomme 2012). De cette façon, les tutorés arriveront à prendre conscience de leurs propres stratégies d'apprentissage et à participer activement à leurs propres apprentissages (Barnier, 1989) tandis que les tuteurs retireront «un bénéfice cognitif personnel» (Barnier, 1989), puisque pour venir en aide aux tutorés, ils auront «à structurer leur pensée, à réorganiser leurs savoirs, ce qui leur permettra de progresser» (Vandomme 2012, p.5). Grâce alors à un ensemble de transactions dynamiques de réflexion et de prise de décision (Bruner, 1983), tutorés et tuteurs arriveront à couvrir le décalage entre leurs savoirs actuels et les accomplissements futurs à réaliser (Vygotsky, 1978).

La réussite d'une expérience tutorielle dépend surtout de la qualité des relations socioaffectives qui se développeront entre tuteurs et tutorés. Le tutoré préfère travailler à l'abri de la critique de l'enseignant, dans une ambiance d'entraide souple et de partenariat. Le tuteur, de son côté, doit respecter les limites de son rôle et ne pas commencer à agir en tant qu'enseignant (Berzin, 2001, p. 128). Dans l'étude de cas que nous décrivons par la suite, l'importance des rapports socioaffectifs surgit dès le début de l'action.

\section{Le cadre organisationnel}

\subsection{Les groupes des tuteurs et des tutorés}

Pendant le semestre de printemps de l'année académique 2012, une expérience de tutorat a été entreprise auprès de deux groupes d'étudiants : 35 étudiants du 1er cycle composent le groupe des tutorés tandis que 6 étudiants en master 2 sont appelés à assumer le rôle de tuteurs. Les deux groupes d'acteurs de l'expérience même s'ils partageaient la même formation initiale et qu'ils avaient comme intention commune de développer et de consolider leur compétence pédagogique, avaient un bagage cognitif différent, vu que les étudiants du $1^{\text {er }}$ cycle se trouvaient à la troisième année de leurs études et que ceux du $2^{\mathrm{e}}$ cycle étaient vers la fin de leurs études postuniversitaires ( $5^{\mathrm{e}}$ année). Ainsi, le rapprochement des contenus des cours, d'un côté, et le décalage cognitif des deux groupes d'étudiants impliqués, de l'autre, ont permis de prévoir un dispositif d'entraide. C'est pourquoi les étudiants de master, en tant qu'enseignants novices, ont été désignés pour guider les étudiants du $1^{\text {er }}$ cycle - futurs enseignants - dans la réalisation de deux devoirs comptant pour leur évaluation finale au cours. De cette façon, les interactions enseignant-savoir-apprenants ont été multipliées, ouvrant de nouveaux canaux de communication dans le groupe même des étudiants.

2 Le tutorat par les pairs est déjà appliqué dans la périphérie d'Athènes (Grèce) à la 2école Primaire d'Aghioi Théodoroi (Enseignante responsable : P. Chaloulou) et au 2e Gymnase d'Aspropyrgos (Enseignante responsable: A. Giannatou). 


\subsection{Les cours impliqués}

L'action tutorielle a été promue au sein de deux cours optionnels, le premier faisant partie du programme d'études du 1er cycle et le deuxième figurant dans la liste des cours du master conjoint franco-hellénique en didactique des langues ${ }^{3}$. Plus particulièrement, il s'agit du cours «Pratiques de classe pour l'enseignement du français langue étrangère » (1er cycle) et du cours «Apprentissage et interaction théâtrale» du $2^{\mathrm{e}}$ cycle, réciproquement. Les deux cours ont été choisis en tant que «lieu de rencontre» de ces deux groupes d'étudiants en raison de la convergence de leurs contenus, le premier étant un cours d'initiation à l'apprentissage du FLE au moyen du jeu dramatique et le deuxième, un cours d'approfondissement sur les pratiques théâtrales, dramatiques et ludiques appliquées au cours de langues. Les contenus de ce nouveau cours comportaient des éléments du cours du $1^{\text {er }}$ cycle et de celui du $2^{\mathrm{e}}$ cycle. En d'autres mots, les deux cours impliqués ont été fusionnés en un seul.

Chaque séance didactique était divisée en deux étapes : la première portait sur la réalisation d'une activité créative ou ludique, inspirée des pratiques théâtrales, et la deuxième était consacrée à un retour réflexif sur l'activité réalisée. Les participants avaient la possibilité, d'un côté, de se mettre à la place de l'apprenant du français langue étrangère et, de l'autre, de réfléchir en tant qu'enseignants sur les points faibles ou forts des activités réalisées ainsi que sur les fondements théoriques des actions menées. De cette façon, le cours avait une double intention pédagogique : d'une part, promouvoir un apprentissage fondé sur le vécu et l'expérience et, d'autre part, favoriser une réflexion concrète sur l'efficacité de l'enseignement proposé.

\subsection{Les participants}

De ce qui précède, il s'ensuit que le bagage cognitif des étudiants impliqués variait de façon considérable : les étudiants du $1^{\text {er }}$ cycle, ayant suivi les cours des deux premières années de leurs études, disposaient d'un bagage cognitif plutôt frêle et déficient non seulement parce que leur premier contact avec la didactique des langues s'était effectué justement pendant le semestre d'hiver de la troisième année de leur cursus ${ }^{4}$, mais surtout parce que la plupart d'entre eux n'avaient jamais enseigné. À l'opposé, les étudiants du $2^{\mathrm{e}}$ cycle faisaient preuve d'une supériorité cognitive parce qu'ils s'étaient déjà confrontés aux fondements théoriques de la didactique des langues au sein du master, mais aussi parce que leur expérience professionnelle, plus ou moins signifiante, en tant qu'enseignants, les aidait à associer plus facilement la théorie à la pratique. En tout cas, l'intention et les attentes de tous les participants au cours se focalisaient sur l'amélioration de leur compétence pédagogique. Les deux groupes d'étudiants formaient, alors, une équipe hétérogène tant au niveau numéral - 32 étudiants du $1^{\text {er }}$ cycle contre 6 étudiants du $2^{e}$ cycle - qu'au niveau cognitif puisque le niveau de connaissances des étudiants postuniversitaires était évidemment plus élevé que celui des étudiants en licence. La contribution, alors, de chaque participant dépendait de ses connaissances et expériences. De cette façon, les étudiants du 1er cycle, s'identifiant davantage avec le rôle de l'apprenant, donnaient des informations intéressantes sur l'activité réalisée, ce qui aidait les étudiants en master à réfléchir de façon plus critique sur l'efficacité des pratiques enseignantes. Par extension, les remarques des étudiants postuniversitaires aidaient les étudiants du 1er cycle à s'imaginer plus facilement à la place de l'enseignant tandis que les discussions portant sur le degré d'efficacité de l'activité menée permettaient aux étudiants de $1^{\text {er }}$ cycle de se mettre aussi à la place de l'enseignant.

\subsection{L'objectif du cours commun}

Malgré le décalage cognitif des deux groupes, le but final de l'expérience restait commun : il s'agissait d'impliquer les étudiants dans un apprentissage dynamique, coopératif et, en grande partie, autogéré. Même si les contenus du cours étaient les mêmes (activités inspirées du jeu dramatique pour l'apprentissage du FLE) pour les deux groupes, les rôles et les fonctions des participants étaient bien distincts en tant que tuteurs et tutorés. Le cadre de travail proposé était censé amener les participants à s'impliquer et à prendre des décisions concernant leur propre apprentissage. En même temps, tous les participants, en tant que futurs ou novices enseignants, auraient la possibilité de se mettre en contact avec un type d'enseignement où l'enseignant s'effacerait en faveur de la mobilisation de l'enseigné. De cette façon, le cours les inciterait à s'essayer eux-mêmes dans des pratiques enseignantes émancipatrices afin d'élargir leurs expériences pédagogiques.

3 Le master est offert par I'Université d'Athènes (Grèce) et l'Université d'Angers (France) depuis 2011 et est intitulé «École et Plurilinguisme. Parcours Professionnel : Enseignants de langues en Europe : formation à la diversité linguistique et culturelle des publics scolaires ». II s'adresse à des diplômés des Facultés des Langues recrutés en Grèce et en France.

$4 \quad$ Curriculum du Département de Langue et Littérature Françaises (2012-13), disponible sur : http://www.frl.uoa.gr/fileadmin/frl.uoa.gr/uploads/ gallika/anakoinosis/Guide_2012-13.pdf (consulté le 20 février 2014) 


\subsection{L'évaluation finale}

L'insertion du tutorat au cours avait comme enjeu la réalisation des deux devoirs. Le premier consistait en la rédaction d'un texte théorique de 2000 mots environ traitant une notion-clé de l'apprentissage interactif (par exemple, la motivation, l'implication, le ludique, etc.) tandis que le devoir pratique portait sur l'organisation et la réalisation en classe d'une activité d'apprentissage conçue selon les principes du jeu dramatique en classe de langue. Les critères d'évaluation pour les deux groupes (tutorés et tuteurs) se différenciaient selon leur statut e,t conséquemment, en fonction de leur niveau de connaissances et des compétences à développer pendant l'expérience du tutorat.

De cette manière, les tutorés devaient, d'un côté, s'initier à la rédaction d'un texte portant sur une question scientifique à l'aide de sources bibliographiques et, de l'autre, concevoir et réaliser en classe une activité inspirée du jeu dramatique. La réalisation de l'activité en classe donnerait la possibilité aux participants d'agir en tant qu'enseignants.

De leur côté, les tutorés devaient guider les étudiants dans la rédaction du devoir théorique et la planification de l'activité. Pour ce faire, ils étaient censés leur fournir toutes les informations nécessaires portant sur les normes de rédaction d'un texte scientifique et les aider à proposer une activité ludique, faisable et originale.

Tuteurs et tutorés seraient évalués en fonction de la qualité des devoirs réalisés.

Plus précisément, pour le groupe des tuteurs, on évaluerait la pertinence de leur soutien à partir de : a) la pertinence des sujets choisis (aussi bien pour le devoir théorique que pour le devoir pratique, b) la conformité de leurs explications concernant les principes de conception d'une activité ludique, et c) l'efficacité de leurs explications portant sur les normes de rédaction d'un texte scientifique.

Pour les tutorés, leurs deux devoirs seraient jugés sur la base : a) de l'adéquation du sujet du devoir théorique, b) de la faisabilité et l'originalité de l'activité proposée, et c) de l'application des normes de rédaction d'un texte scientifique.

\section{La mise en place}

\subsection{Les conditions du déroulement}

L'expérience a duré tout au long du semestre de printemps à raison d'un cours de trois heures par semaine. Pour les six premières séances didactiques, la distinction entre tuteurs et tutorés n'était pas visible. Il s'agissait d'une période de (re) connaissance mutuelle et de coexistence, ce qui permettait aux membres des deux groupes de se bâtir une nouvelle relation, celle de tuteur-tutoré.

À partir du moment où les devoirs pour l'évaluation finale ont été annoncés - vers la fin du deuxième mois du semestre l'enseignante a expliqué les conditions de l'action de tutorat qui allait avoir lieu entre les deux groupes d'étudiants, en vue de la réalisation des devoirs finaux. On a aussi expliqué les obligations de chaque groupe et de chaque membre du groupe : les tutorés avaient à coopérer avec le tuteur de leur groupe pour la coordination des devoirs à réaliser tandis que les tuteurs devaient guider les tutorés de leur groupe en leur fournissant toutes les informations et rectifications nécessaires. Pour ce faire, des heures supplémentaires de consultation ont été organisées par le tuteur de chaque groupe.

Les étudiants du $1^{\text {er }}$ cycle ont été répartis en groupes de 4-5 personnes. Un des six étudiants du $2^{\mathrm{e}}$ cycle a été désigné comme tuteur pour chaque groupe d'étudiants. On a aussi précisé que chaque étudiant pouvait s'adresser au tuteur de son groupe pour toute question ou difficulté qu'il envisageait, ce qui ne l'empêcherait pas pour autant de s'adresser également à l'enseignante directement. Le groupe des tuteurs, de leur côté, se trouvait en coopération étroite avec l'enseignante, tout d'abord pour planifier leurs actions et ensuite, chaque fois que cela s'avérait nécessaire, pour reconsidérer ou réajuster leurs interventions tutorielles. En dépit de ces séances de discussion et de consultation avec l'enseignante, les tuteurs étaient censés intervenir en pleine autonomie auprès des étudiants de leur groupe. En outre, les décisions portant sur les devoirs étaient le produit d'un consensus entre les tutorés eux-mêmes et leur tuteur.

\subsection{Les premières réactions}

L'hétérogénéité des groupes a causé des problèmes au début de l'action; les étudiants du 1 er cycle semblaient être plus à l'aise avec l'enseignante qu'avec les tuteurs. Par conséquent, les étudiants des deux groupes (tuteurs et tutorés) donnaient l'impression d'éprouver une certaine méfiance envers cette nouvelle pratique. C'est pour cette raison peut-être que, pendant 
le cours, les étudiants du $1^{\text {er }}$ cycle préféraient travailler entre eux et non pas avec les étudiants du $2^{\mathrm{e}}$ cycle et vice versa. Cette pratique a conditionné les échanges entre tuteurs et tutorés qui, au début, étaient très hésitants et limités. Ce climat a commencé à être renversé après l'intervention didactique d'une tutrice qui a réalisé un cours à la place de l'enseignante. En outre, la réalisation d'une activité théâtrale par une actrice invitée au cours a contribué à ce que les rapports des participants deviennent moins méfiants. Le renversement de cette première méfiance a été, d'abord, reflétée sur l'image spatiale de la classe : les étudiants du $1^{\text {er }}$ cycle se sont déplacés et se sont assis à côté des étudiants du $2^{\mathrm{e}}$ cycle — et peu à peu, des échanges plus spontanés ont commencé à se nouer entre les deux groupes. Le rôle des étudiants du $2^{\mathrm{e}}$ cycle a commencé à se modifier à partir du moment où ils se sont mis à conseiller ceux du $1^{\text {er }}$ cycle en vue de la réalisation des devoirs.

\subsection{Le bilan de l'expérience}

Les résultats obtenus aux deux devoirs constitueraient les premiers indices d'efficacité de l'intervention tutorielle. En outre, une deuxième étape d'évaluation de l'efficacité du tutorat a été prévue à la fin du semestre à la base de deux questionnaires, un pour les tuteurs et un deuxième pour les tutorés. Ces questionnaires ont été conçus pour mettre en évidence les représentations des participants sur les avantages et les difficultés du tutorat, sa valeur didactique et pédagogique ainsi que sur leur disposition, après l'expérience, d'utiliser à l'avenir le tutorat en tant que pratique enseignante.

\subsection{Les résultats aux devoirs finaux}

Les activités ludiques proposées par les étudiants étaient toutes bien conçues, révélatrices d'un travail préalable, important et créatif. Par ailleurs, les tuteurs de tous les groupes n'ont pas hésité à participer en termes égaux avec les tutorés à la réalisation de l'activité proposée, même si cela ne faisait pas partie de leurs obligations, ce qui dévoile l'esprit de convivialité développé au sein de chaque groupe ainsi que l'ambiance de sécurité et d'acceptation qui s'était substituée au sentiment de méfiance initiale.

De leur côté, les tutorés avaient l'air de se sentir bien à l'aise dans leur rôle d'enseignant lors de la mise en place de l'activité proposée. La complicité partagée par les membres de chaque groupe était bien évidente lors de la réalisation de l'activité en classe.

Pour ce qui est des devoirs théoriques, les membres d'un seul groupe se sont éloignés de la consigne de la tâche, à savoir l'exploitation d'une notion-clé de l'apprentissage par le jeu dramatique. Le tuteur du groupe a reconnu que ses explications erronées avaient amené les membres de son groupe à concevoir différemment le contenu du devoir. Les devoirs théoriques des autres groupes étaient bien conçus et suivaient de près les normes de rédaction d'un devoir de ce type.

\subsection{L'expérience vue par les tutorés}

\section{a) L'évolution relationnelle}

La plupart des étudiants du $1^{\text {er }}$ cycle avouent qu'au début du semestre considéraient que leurs relations avec ceux du $2^{\mathrm{e}}$ cycle étaient «hésitantes» ou même «neutres», "conventionnelles» ou «fragmentaires». Seul un tiers des participants les caractérisent comme «bonnes» dès le début. De façon générale, ce qui les empêchait de s'ouvrir aux étudiants de master, c'était surtout, leur supériorité cognitive et leur aisance à parler français. Ils avaient peur que leur production orale ne dévoile une déficience dans leurs compétences langagières. Alors, ils préféraient rester muets ou s'exprimer de façon très laconique. En outre, la différence d'âge et l'attitude des étudiants en master ne leur permettaient pas de se sentir à l'aise avec eux, ce qui les rendait encore plus réticents. Toutefois, pendant le tutorat, ils reconnaissent que leurs relations sont devenues plus harmonieuses. À la fin du tutorat, la majorité des étudiants du $1^{\text {er }}$ cycle trouvent que leurs relations avec leurs tuteurs étaient amicales et même «chaleureuses» et «exceptionnellement bonnes». Un seul étudiant a déclaré qu'il avait des problèmes de communication avec son tuteur, dès le début jusqu'à la fin de l'expérience.

\section{b) Les sources de blocage}

Les étudiants précisent qu'ils n'avaient pas d'expérience antérieure de tutorat et qu'au début la perspective de participer à un cours dont ils ignoraient les règles les a rendus plutôt stressés et inquiets. En outre, la présence des étudiants de master qui venaient de rentrer de leur semestre en France et qui pouvaient s'exprimer très bien en français était un autre facteur qui avait un impact négatif sur leur comportement. De cette façon, ils ne pouvaient pas bien comprendre leur rôle dans ce cours 
dont ils croyaient que l'objectif et les contenus dépassaient largement leurs possibilités. Ainsi, ils ne pouvaient pas se faire une idée précise de leur statut au cours ni de la contribution des étudiants de master. Paradoxalement, ils trouvent que la présence de leurs confrères de master était «utile», «importante», "signifiante» ou même «nécessaire». En outre, ils croient que les étudiants de master s'assuraient à ce que leur présence soit « discrète», ce qui les a aidés petit à petit à se sentir plus à l'aise. Une fois le tutorat mis en place, ils trouvent que le rôle consultatif des tuteurs, a été «très utile», «important», «nécessaire » ou tout simplement «satisfaisant». II y a eu deux étudiants qui n'étaient pas du même avis : I'un a noté que les tuteurs "devaient être mieux informés sur l'objet et les modalités d'évaluation», et l'autre a constaté que le rôle consultatif de son tuteur était «médiocre à cause des tensions qui existaient dans le groupe». En général, le tutorat est envisagé plus positivement vers la fin de l'expérience qu'au début. Tous les étudiants, sauf un, le caractérisent comme une expérience «très utile», «intéressante» et «totalement réussie». II faut noter que l'étudiant qui avait décrit le rôle consultatif de son tuteur comme "médiocre à cause des tensions qui existaient dans le groupe» a trouvé l'expérience du tutorat «intéressante». À la question : "est-ce que vous utiliseriez, en tant que professeurs, le tutorat entre élèves à l'école?», tous les étudiants ont de nouveau répondu d'une manière positive, sauf un. Il est intéressant de noter que l'étudiant qui avait décrit la présence des étudiants du $2^{\mathrm{e}}$ cycle comme «intéressante» a dit qu'il «ne serait pas facile» d'adopter une telle démarche à l'école parce que les élèves n'ont pas I'habitude de travailler de cette façon. Une autre étudiante a aussi évoqué les éventuelles réactions de la direction de l'école ou même des parents envers des pratiques de ce type qui, selon elle, s'éloigne du cours traditionnel. Paradoxalement, l'étudiant qui jugeait le tutorat comme «médiocre à cause des tensions qui existaient dans le groupe» a déclaré qu'il opterait pour cette démarche en tant qu'enseignant. Ces remarques contradictoires révèlent peut-être les hésitations des étudiants qui se sentent partagées entre leurs propres représentations de ce qui est un cours à l'école et de leur propre expérience du tutorat. En tout cas, elles sont surtout liées à des hésitations, des résistances et des méfiances tout à fait justifiables à cause du manque d'expérience préalable.

\section{c) Les apports de l'expérience}

En dépit de leurs hésitations, les étudiants croient que le rôle médiateur des tuteurs les a aidés à mieux rédiger le devoir théorique et à mieux concevoir l'activité du jeu dramatique. Ils croient que l'expérience de leurs tuteurs en tant qu'enseignants leur a été très utile. En outre, ils précisent qu'en tant que futurs enseignants ils ont pu comprendre comment expliquer et comment faire face aux difficultés de leurs élèves. De fait, ils se sentaient plus préparés au métier d'enseignant. De cette façon, presque tous croient avoir amélioré leur compétence pédagogique surtout grâce à leur familiarisation avec les activités du jeu dramatique. Néanmoins, ils se montrent hésitants quant à une éventuelle application du tutorat en tant qu'enseignants à l'école parce qu'ils croient que ce type d'activités et d'apprentissage ne font pas partie des pratiques habituellement promues à l'école grecque. En outre, ils se sont prononcés très sceptiques envers des problèmes de discipline pendant la réalisation d'un cours interactif. Ils reconnaissent pour autant que les échanges avec leurs camarades les ont aidés à apprendre de façon plus agréable et facile.

Pour ce qui était de la rédaction du devoir théorique, la majorité des étudiants croient qu'ils ont réussi à maîtriser les normes de rédaction d'un dossier/devoir grâce à leur collaboration avec les étudiants du $2^{\mathrm{e}}$ cycle. II faut noter, tout de même, que cette opinion n'est pas partagée par tous; il y a un étudiant qui déclare que, même s'il pense qu'il a acquis beaucoup de connaissances sur les pratiques de classe, le tutorat ne l'a pas aidé à améliorer son savoir-faire en ce qui concerne la rédaction d'un devoir. Concernant la conception et la réalisation de l'activité, la plupart des étudiants sont enthousiastes de la collaboration avec leur tuteur et des résultats obtenus. Les membres d'un seul groupe ont eu beaucoup de difficultés de coopération avec leur tuteur, ce qui pour autant n'a pas nui à la qualité de l'activité proposée. Ils soulignent néanmoins que les difficultés dans la collaboration et la prise de décision commune ont conditionné les rapports du groupe qui n'étaient pas du tout harmonieux. En fin de compte, le bilan général de l'expérience s'avère positif pour la majorité des étudiants. Trois tutorés de groupes différents ont même déclaré qu'ils souhaitaient continuer leur collaboration avec leurs tuteurs au sein d'autres cours. 


\section{a) Une attitude de méfiance}

Au début du semestre, les étudiants du $2^{e}$ cycle se montrent eux aussi méfiants face aux conditions du déroulement du cours ainsi qu'à ses objectifs. À cause des différents niveaux cognitifs des deux groupes, ils se sentaient assez confus au début de l'action. Plus précisément, ils ne pouvaient pas comprendre comment ce cours allait leur fournir des connaissances nouvelles puisqu'ils allaient travailler en parallèle avec les étudiants du $1^{\text {er }}$ cycle. De surcroît, ils ne se sentaient pas assez positifs envers un cours qui solliciterait leur exposition au public et surtout à un public composé d'étudiants plus jeunes et moins qualifiés qu'eux.

Les étudiants finissent par constater que cette attitude a commencé à changer peu à peu, surtout grâce à la contribution du jeu dramatique au changement des rapports entre les participants. C'est pourquoi ils sont très positifs envers l'apport des pratiques théâtrales et du jeu dramatique dans le développement d'une compétence sociale et d'une facilité de s'exprimer de façon plus spontanée et rassurée en public. Ils trouvent que cette compétence d'exposition publique est très utile en général aux enseignants. En revanche, ils avouent qu'au début de l'expérience ils ne s'attendaient pas à ce que le tutorat puisse les aider à développer leur compétence pédagogique. Ils se faisaient, d'ailleurs, une représentation plutôt positive de leur compétence pédagogique.

\section{b) Une évolution relationnelle}

En ce qui concerne leurs relations avec les tutorés, les étudiants-tuteurs reconnaissent qu'il s'agit du domaine qui était le plus difficile à gérer au début, mais qui a le plus évolué pendant l'expérience. Ils y distinguent trois phases d'évolution :

- Au début, ils admettent qu'ils se croyaient eux-mêmes supérieurs aux autres étudiants, surtout au niveau cognitif. Cette conviction a renforcé leurs difficultés d'adaptation aux conditions du cours; c'est pourquoi, pendant les premières séances didactiques, ils se sont montrés peu ouverts envers les autres étudiants. Ils préféraient s'asseoir tous ensemble un peu à l'écart des autres étudiants, marquant de la sorte leur méfiance, mais aussi leur supériorité.

- Au fur et à mesure que le cours avançait, les relations des deux groupes se sont transformées en des relations de coopération et de réciprocité. Ce changement s'est vite reflété même sur la disposition sociospatiale de la classe, puisque les étudiants-tuteurs ont commencé à côtoyer les étudiants de licence.

- À la fin du semestre, ils croient avoir créé des liens affectifs considérables avec les étudiants du 1er cycle, allant jusqu'à ce qu'ils nouent des relations amicales avec les tutorés.

En tout cas, cette évolution dans les relations tuteurs-tutorés, a été permise, selon eux, grâce, au jeu dramatique, d'un côté, et au tutorat, de l'autre, puisqu'ils avaient la possibilité de coopérer avec les autres étudiants et de valider ainsi leur supériorité cognitive. Ils insistent en outre sur le fait que ce rapprochement ne pourrait être effectué que de façon souple et progressive et qu'il fallait du temps pour que les membres des deux groupes puissent surmonter leurs propres habitudes et attitudes. Le rôle de l'enseignante se caractérise comme «neutre», puisqu'il se limitait au soutien général et, parfois, à l'apaisement des différences et des problèmes entre les tuteurs et les tutorés. Peu souvent et surtout au début de l'action, ils se sont vus obligés de s'adresser à l'enseignante pour gérer un problème de coopération ou d'autre nature au sein de leur groupe.

\section{c) Un écart cognitif résorbé}

En ce qui concerne la différence du niveau cognitif, les tuteurs croient que, malgré leur méfiance première envers l'efficacité de cet enseignement parallèle dirigé, ils ont fini par le considérer comme une stratégie pédagogique et didactique qui permettait aux participants d'apprendre et d'approfondir en pleine convivialité et coopération. Ils ont aussi souligné que ce type d'enseignement les incitait constamment à revenir sur leurs propres connaissances et compétences, à les reconsidérer et à les réajuster pour mieux atteindre leur but. Leur contact avec les tutorés les a aussi aidés à mieux comprendre les difficultés 
que les étudiants en licence envisageaient. Ils découvraient avec grande surprise que les difficultés des tutorés n'étaient pas les mêmes que les leurs: ce qui pour eux était clair pouvait présenter une difficulté insurmontable pour les tutorés. Cet aspect du tutorat les a amenés à réfléchir sur les origines des difficultés et des démarches adoptées pour y faire face. Au sein même de chaque groupe de tutorés, ces difficultés étaient bien différentes. Ils avaient alors à opter pour une collaboration collective et aussi individualisée, ce qui, selon eux, les a obligés à trouver des solutions différentes. En fin de compte, malgré les difficultés à gérer une pratique qui leur était en grande partie inconnue, les étudiants-tuteurs caractérisent l'expérience comme un mécanisme de rétroaction, cognitive et attitudinale, qui les poussait sans cesse à améliorer leur tactique en tant que tuteurs. En tant que tels, ils croient que le tutorat les a aidés à apprendre à expliquer quelque chose de façon plus simplifiée et claire en prenant en considération les particularités de celui à qui on s'adressait.

\section{d) Les avantages de l'expérience}

Les étudiants-tuteurs constatent que l'expérience de tutorat leur a aussi offert la possibilité de progresser dans des domaines différents :

- À force d'expliquer, ils finissaient par mieux maîtriser leurs propres connaissances. La plupart d'entre eux soulignent le fait qu'ils avaient pu mieux s'approprier les techniques et les normes de rédaction d'un texte scientifique à force de les expliquer aux tutorés de leur groupe. Leurs connaissances sur ce domaine ont pu, alors, se transformer en un véritable savoir-faire, ce qui leur a été fort utile dans la rédaction du Mémoire et du Rapport du Stage Pratique.

- Sur le niveau pédagogique, leur rôle en tant que tuteurs les obligeait à réfléchir sur le comment corriger ou le comment dire à un tutoré que son travail exigeait une refonte. Ainsi, ils ont dû renoncer à des pratiques plus autoritaires qui ne s'avéraient pas adéquates pour un travail entre pairs. De cette façon, ils croient que leur compétence pédagogique a été davantage développée, vu que le tutorat par pairs mettait en valeur leur supériorité cognitive, mais qu'en même temps, leur rappelait qu'ils étaient tous en train d'apprendre, ce qui les empêchait d'opter pour des pratiques d'explicitation fondées uniquement sur leur supériorité cognitive.

- $\quad$ S'ils ont aussi pris conscience que les relations affectives entre celui qui enseigne et celui qui apprend favorisent l'apprentissage et la coopération et que, par conséquent, l'apprentissage peut être plus effectif si des relations de respect et de mutualité sont nouées.

- Un autre point important pour eux est la socialisation des apprenants. Ils soulignent que les interactions entre tuteurs et tutorés les ont amenés à dépasser leur individualisme et à développer leur compétence sociale, ce qui a contribué à la responsabilisation des participants envers eux-mêmes, mais aussi envers les autres.

- En outre, les étudiants-tuteurs se voient plus préparés à leur rôle d'enseignant à la fin de l'expérience parce qu'ils croient être plus performants et «professionnels».

- Enfin, la plupart d'entre eux soutiennent qu'ils adapteront à l'avenir en tant qu'enseignants le tutorat par les pairs auprès de leurs apprenants surtout pour favoriser l'apprentissage interactif et autonome.

\section{e) Les difficultés rencontrées}

L'ouverture envers l'autre a été, selon les étudiants-tuteurs, le point le plus difficile à surmonter. Les raisons les plus souvent évoquées de cette situation sont le manque d'expérience antérieure ainsi que les représentations figées qu'ils se faisaient du rôle de l'enseignant et de celui de l'apprenant.

Un autre paramètre était la gestion de l'acte tutoriel lui-même de nouveau à cause de l'absence de toute expérience antérieure. Cela les a parfois amenés à gérer maladroitement le temps qu'ils avaient à leur disposition ou à s'adresser de façon peu convaincante aux membres de leur groupe, ce qui parfois rompait ou bloquait la communication. Les conflits ou les malentendus surgis lors de leur tutorat étaient un point difficile à gérer. Au début, ils s'adressaient à l'enseignante pour trouver une solution, mais petit à petit, ils se sont sentis plus sûrs d'eux-mêmes et ils arrivaient à se débrouiller seuls. 
Malgré ces difficultés inévitables, les étudiants-tuteurs croient que l'expérience de tutorat elle-même leur donnait la possibilité de prendre conscience de leurs démarches, de les reconsidérer et, éventuellement, de les réadapter. En général, ils se considèrent plus préparés à enseigner.

\section{Conclusion}

Le cas de tutorat par pairs que nous venons de décrire a su mettre en évidence son apport au développement de l'expérience et de la compétence pédagogiques lors de la formation initiale ou continue des enseignants de langues. Ses bienfaits portent surtout sur l'encouragement de la gestion autonome des apprentissages et l'incitation à la prise de décisions par les formés. En même temps, nous avons pu constater que les résistances éprouvées par les participants envers une forme d'enseignement-apprentissage qui ne faisait pas partie de leurs expériences antérieures étaient bien tangibles et importantes. Nous considérons que ce point qui semble peut-être nuire à l'applicabilité d'une action de tutorat est, en même temps, son point le plus fort parce qu'il dévoile un besoin réel d'insérer des démarches enseignantes reliant davantage la théorie à la pratique. De surcroît, le tutorat par pairs dans la formation des enseignants de langues donne la possibilité aux participants de (re) considérer leurs convictions plus ou moins stéréotypées et préconstruites de ce que c'est d'enseigner et d'apprendre. De cette façon, leur formation peut s'avérer plus efficiente et rentable. Évidemment, l'absence d'expérience préalable par les étudiants impliqués a mis aussi en évidence la rigidité et l'étanchéité qui régissent les relations entre les membres d'un groupe d'étudiants. La mutation des rapports interpersonnels s'est avérée d'ailleurs le point tournant de la réussite du cas étudié. À partir du moment où les participants ont commencé à s'ouvrir les uns aux autres et à se socialiser dans le cadre de l'action, l'enseignement et l'apprentissage promus ont eu un rendement considérable. En général, les difficultés et les contraintes cognitives ou relationnelles imposées par le tutorat, au lieu de bloquer le déroulement et l'accomplissement de l'action, ont pu amener les étudiants à la responsabilisation et à la prise en main d'un enseignement-apprentissage dynamique.

\section{Références bibliographiques}

Barnier, G. (1989, septembre). L'effet-tuteur dans des situations mettant en jeu des rapports spatiaux chez des enfants de 7-8 ans en interactions dyadiques avec des pairs de 6-7 ans. European Journal of Psychology of Education (vol.4), 385-399.

Baudrit, A. (2001). Le tutorat. Richesse d'une méthode pédagogique. Bruxelles : De Boeck Supérieur.

Baudrit, A. (2003). Le tutorat à l'école. Que peuvent faire les élèves tuteurs ?. Carrefours de l'éducation (1), 15,118-134.

Becker, H. (1983). Outsiders : études de sociologie de la déviance. Paris : éditions Métailié.

Berzin, C. (2001, janvier). Interactions de tutelle, développement et apprentissages. Carrefours de l'éducation (11), $120-147$.

Bruner, J. (1983). Le développement de l'enfant: savoir faire savoir dire. Paris : PUF.

Fredy-Planchot, A. (2007, juin). Reconnaître le tutorat en entreprise. Revue française de gestion, 175, 23-32. Repéré à www. cairn.info/revue-francaise-de-gestion-2007-6-page-23.htm. DOI: 10.3166/rfg. 175.23-32

Moussay, S., Étienne, R., \& Méard, J. (2009, janvier-mars). Le tutorat en formation initiale des enseignants : orientations récentes et perspectives méthodologiques. Revue française de pédagogie. 166, 59-69. Repéré à http://rfp.revues.org/1127

Olry-Louis, I. (2009). Les activités communicatives des tuteurs en psychologie du développement et en pratiques à l'université. Recherche et formation, 62, 77-90.

Papi, C. (2013). Le tutorat de pairs dans l'enseignement supérieur : enjeux institutionnels, technopédagogiques, psychosociaux et communicationnels. Paris : L'harmattan.

Rodet, J. (2007). Tutorat à distance, une fonction essentielle. Repéré à http://jacques.rodet.free.fr/ xchroete.htm.

Vandomme, A. (2012). Le tutorat : I'effet-tuteur. Éducation. Repéré à http://dumas.ccsd.cnrs.fr/ dumas00735892/document

Vygotsky, L. (1978). Interaction between learning and development. Mind and Society, 79-91. Cambridge, MA: Harvard University. 